\title{
A note on cut-elimination for classical propositional logic
}

\author{
Gabriele Pulcini ${ }^{1}$
}

Received: 30 April 2021 / Accepted: 21 September 2021 / Published online: 26 November 2021

(c) The Author(s) 2021

\begin{abstract}
In Schwichtenberg (Studies in logic and the foundations of mathematics, vol 90, Elsevier, pp 867-895, 1977), Schwichtenberg fine-tuned Tait's technique (Tait in The syntax and semantics of infinitary languages, Springer, pp 204-236, 1968) so as to provide a simplified version of Gentzen's original cut-elimination procedure for firstorder classical logic (Gallier in Logic for computer science: foundations of automatic theorem proving, Courier Dover Publications, London, 2015). In this note we show that, limited to the case of classical propositional logic, the Tait-Schwichtenberg algorithm allows for a further simplification. The procedure offered here is implemented on Kleene's sequent system G4 (Kleene in Mathematical logic, Wiley, New York, 1967; Smullyan in First-order logic, Courier corporation, London, 1995). The specific formulation of the logical rules for G4 allows us to provide bounds on the height of cut-free proofs just in terms of the logical complexity of their end-sequent.
\end{abstract}

Keywords Classical propositional logic $\cdot$ Sequent calculus $\cdot$ Cut elimination

Mathematics Subject Classification 03F05 Cut-elimination and normal-form theorems

\section{Introduction}

In [5], Schwichtenberg fine-tuned Tait's technique [7] so as to provide a simplified version of Gentzen's original cut-elimination procedure, which notoriously requires a complex induction on a certain lexicographic order [2]. In particular, Schwichtenberg showed that termination of the cut-elimination procedure can be achieved by resorting to two independent inductions on $\omega$. The Reduction Lemma is proved by induction on the sum of the heights of the two derivations delivering the premises of the cut-

The author would like to thank Enrico Moriconi for his careful reading of the final version of the paper.

$凶$ Gabriele Pulcini

gabriele.pulcini@uniroma2.it

1 Dipartimento di Studi Letterari, Filosofici e di Storia dell'Arte, Università di Roma “Tor Vergata", Rome, Italy 
application under consideration [5, Lemma 2.6, p. 874] and the final Hauptsatz is proved by induction on the cut-rank of the whole proof [5, Theorem 2.7, p. 875].

In this note we show that, limited to the case of classical propositional logic, cutelimination allows for a further simplification. As a matter of fact, the proof of Lemma 4 (our Reduction Lemma) is simply led by cases, whereas Theorem 5 (the Hauptsatz) is proved by a double induction on the cut-size of proofs and on the number of maximal cut-applications. The size of a cut-application is just defined as the number of connectives occurring in one of its premises. Accordingly, the cut-size of a proof $\pi$ is defined as the supremum of all the cut-sizes relating to $\pi$.

The algorithm proposed in this note is tailored on the sequent system GS4, the one-sided formulation à la Tait of Kleene's G4 $[3,6]$. The procedure heavily relies on the fact that, for any non-atomic formula $A$, if the sequent $\vdash \Gamma, A$ is provable in GS4, then it is also provable by means of a particular proof in which $A$ occurs as the principal formula in the last inference step (Lemma 3 ). The main advantage of dealing with Kleene's system GS4 lies in the fact that the height of cut-free proofs turns out to be bounded by the number of occurrences of logical connectives in their end-sequent (Theorem 6). Moreover, we prove that any two cut-free proofs ending in the same sequent have always the same height (Theorem 7).

\section{Preliminary notions and results}

Following [7], we limit ourselves to considering only two connectives: conjunction $(\wedge)$ and disjunction $(\vee)$. In formal languages $\grave{a}$ la Tait, negation comes as primitive on atomic sentences $\mathbf{A T}=\{p, \bar{p}, q, \bar{q}, \ldots\}$ and it extends to compound formulas by means of the following equivalences:

$$
\overline{\bar{A}} \Leftrightarrow A \quad \overline{A \wedge B} \Leftrightarrow \bar{A} \vee \bar{B} \quad \overline{A \vee B} \Leftrightarrow \bar{A} \wedge \bar{B}
$$

The set $\mathcal{F}$ of well-formed formulas is defined accordingly:

$$
\mathcal{F}::=\mathbf{A T}|\mathcal{F} \wedge \mathcal{F}| \mathcal{F} \vee \mathcal{F} \text {. }
$$

Logical contexts $\Gamma, \Delta, \ldots$ are taken to be multisets of formulas from $\mathcal{F}$. As usual, we write $\Gamma, A$ and $\Gamma, \Delta$ to mean the two multisets $\Gamma \uplus[A]$ and $\Gamma \uplus \Delta$, respectively. We write $\{\Gamma\}$ to indicate the set collecting the elements of $\Gamma$.

We call GS4 the one-sided version of Kleene's sequent system G4 whose rules are displayed in Fig. $1[1,3,4,6]$. The height $h(\pi)$ of a proof $\pi$ is given by the number of sequents occurring in one of its longest branches. A subproof $\delta$ of a proof $\pi$ is said to be direct in case $\delta$ ends in one of the premises of $\pi$ 's last inference. Moreover, we recall that any application of the logical rules displays a principal formula in the conclusion: the formula whose principal connective has been introduced by the very inference step under consideration.

Definition 1 The complexity $\mathcal{C}(A)$ of a formula $A$ is given by the number of occurrences of logical connectives in $A$. More formally: $\mathcal{C}(A)=0$, for any $A \in \mathbf{A T}$, and 


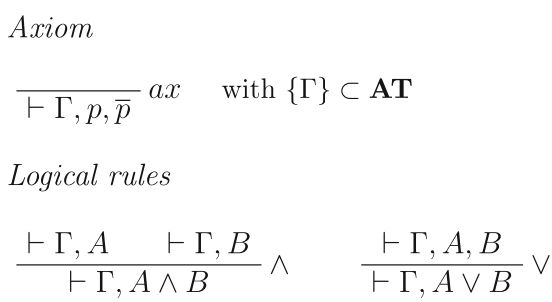

Fig. 1 The rules of the sequent calculus GS4

$\mathcal{C}(A \wedge B)=\mathcal{C}(A \vee B)=\mathcal{C}(A)+\mathcal{C}(B)+1$. For any multiset $\Gamma=\left[A_{1}, A_{2}, \ldots, A_{n}\right]$, we set $\mathcal{C}(\Gamma)=\mathcal{C}\left(A_{1}\right)+\mathcal{C}\left(A_{2}\right)+\cdots+\mathcal{C}\left(A_{n}\right)$.

Remark 1 For any multiset of formulas $\Gamma, C$, we have $\mathcal{C}(\Gamma, C)=\mathcal{C}(\Gamma, \bar{C})$.

Observe that, in the specific formulation adopted here, instances of the $a x$-rule must be clauses, i.e., sequents in which only atomic formulas from AT are displayed. The next proposition shows that such a linguistic restriction does not affect provability.

Proposition 1 GS4 proves the sequent $\vdash \Gamma, p, \bar{p}$, for any multiset of formulas $\Gamma$, and any $p \in \mathbf{A T}$.

Proof We proceed by induction on $\mathcal{C}(\Gamma)$. If $\mathcal{C}(\Gamma)=0$, then $\vdash \Gamma, p, \bar{p}$ is already an instance of the $a x$-rule. As for $\mathcal{C}(\Gamma)>0$, we distinguish two cases:

- $\Gamma=\Gamma^{\prime}, A \wedge B$. By inductive hypothesis, there are two GS4-proofs $\delta$ and $\rho$ ending in $\vdash \Gamma^{\prime}, A, p, \bar{p}$ and $\vdash \Gamma^{\prime}, B, p, \bar{p}$, respectively. The two proofs $\delta$ and $\rho$ can be then composed by means of an application of the $\wedge$-rule so as to finally get the conclusion $\vdash \Gamma^{\prime}, A \wedge B, p, \bar{p}$.

- $\Gamma=\Gamma^{\prime}, A \vee B$. Similar to the previous case.

Below, we recall the well-known fact that the structural rule of Weakening is admissible in GS4 (cfr, for instance, [5, Lemma 2.3.1, p. 873]):

Lemma 2 (Weakening admissibility) If GS4 proves $\vdash \Gamma$, then it also proves the sequent $\vdash \Gamma$, A, for any formula $A$.

Proof Let $\pi$ be a GS4-proof ending in $\vdash \Gamma$. Once the formula $A$ is uniformly added to all the sequents occurring in $\pi$, each of $\pi$ 's top sequents $\vdash \Gamma, p, \bar{p}$ is turned into the sequent $\vdash \Gamma, A, p, \bar{p}$ which is, by Proposition 1 , provable.

Notation Given a GS4-proof $\pi$ of $\vdash \Gamma$ and a formula $A$, we denote with $\mathcal{W}(\pi, A)$ the GS4-proof of $\vdash \Gamma, A$ obtained from $\pi$ according to the procedure employed in the proof of Lemma 2. If $A \in \Gamma$, then $\mathcal{W}(\pi, A)=\pi$.

The following lemma states a peculiar property of the GS4 system which will prove crucial to attain the results proposed in the next section. Such a property comes as a byproduct of the fact that GS4 logical rules are all reversible in the sense that provability of the conclusion always implies provability of the premise(s) (cfr. [5, Lemma 2.5, p. 873]). 
Lemma 3 (Height-preserving permutability) Assume there is a GS4-proof $\pi$ of $\vdash \Gamma, A$ with $\mathcal{C}(A)>0$. The sequent $\vdash \Gamma, A$ is also provable by means of a proof $\rho$ such that: (i) the formula A occurs as principal in $\rho$ 's last inference, and (ii) $h(\pi)=h(\rho)$.

Proof If $\mathcal{C}(\Gamma)=0$, then $\pi$ 's last rule must be already the one introducing $A$ 's principal connective and so $\rho=\pi$. Otherwise, we proceed by showing that any proof $\pi$ of $\vdash \Gamma, A$ can be turned into a proof $\rho$ of $\vdash \Gamma, A$ having the desired form, simply by permuting downwards along $\pi$ the specific instance of the logical rule introducing $A$ 's principal connective. The proof is led by induction on $\mathcal{C}(\Gamma, A)$. We shall be considering the following four possible situations.

- $A \equiv B \wedge C$ and $\pi$ 's last rule is a $\wedge$-rule. Let $D \wedge E$ be the formula occurring as principal in $\pi$ 's last inference, and $\pi_{1}$ and $\pi_{2}$ the two direct subproofs of $\pi$ ending in $\vdash \Gamma, B \wedge C, D$ and $\vdash \Gamma, B \wedge C, E$, respectively. By inductive hypothesis, there is a proof $\pi^{\prime}$ shaped as displayed below, such that $h\left(\pi_{1}\right)=\max \left(h\left(\pi_{\langle 1,1\rangle}\right), h\left(\pi_{\langle 1,2\rangle}\right)\right)+$ 1 and $h\left(\pi_{2}\right)=\max \left(h\left(\pi_{\langle 2,1\rangle}\right), h\left(\pi_{\langle 2,2\rangle}\right)\right)+1$.

$$
\begin{array}{cccc}
\pi_{\langle 1,1\rangle} & \pi_{\langle 1,2\rangle} & \pi_{\langle 2,1\rangle} & \pi_{\langle 2,2\rangle} \\
\vdots & \vdots & \vdots & \vdots \\
\frac{\vdash \Gamma, B, D}{\vdash \Gamma, B \wedge C, D} \vdash \Gamma, C, D & \frac{\vdash \Gamma, B, E}{\vdash \Gamma, B \wedge C, E} \wedge
\end{array}
$$

The proof $\pi^{\prime}$ can be then rearranged into the proof $\rho$ reported below, simply by interchanging the two final applications of the logical rules.

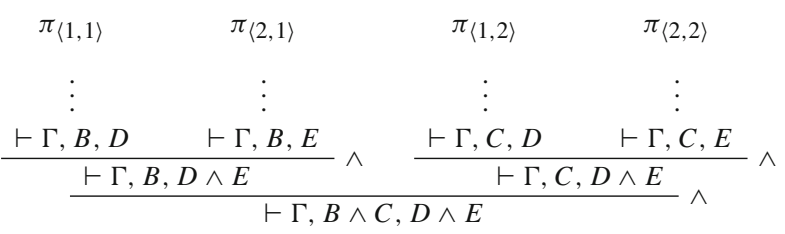

We finally observe that:

$$
\begin{aligned}
h(\pi) & =\max \left(h\left(\pi_{1}\right), h\left(\pi_{2}\right)\right)+1= \\
& =\max \left(\max \left(h\left(\pi_{\langle 1,1\rangle}\right), h\left(\pi_{\langle 1,2\rangle}\right)\right)+1, \max \left(h\left(\pi_{\langle 2,1\rangle}\right), h\left(\pi_{\langle 2,2\rangle}\right)\right)+1\right)+1 \\
& =\max \left(h\left(\pi_{\langle 1,1\rangle}\right), h\left(\pi_{\langle 1,2\rangle}\right), h\left(\pi_{\langle 2,1\rangle}\right), h\left(\pi_{\langle 2,2\rangle}\right)\right)+2 \\
& =\max \left(\max \left(h\left(\pi_{\langle 1,1\rangle}\right), h\left(\pi_{\langle 2,1\rangle}\right)\right)+1, \max \left(h\left(\pi_{\langle 1,2\rangle}\right), h\left(\pi_{\langle 2,2\rangle}\right)\right)+1\right)+1 \\
& =h(\rho)
\end{aligned}
$$

- $A \equiv B \vee C$ and $\pi$ 's last rule is a $\wedge$-rule. Let $D \wedge E$ be the formula occurring as principal in $\pi$ 's last inference, and $\pi_{1}$ and $\pi_{2}$ the two direct subproofs of $\pi$ ending in $\vdash \Gamma, B \vee C, D$ and $\vdash \Gamma, B \vee C, E$, respectively. By inductive hypothesis, there is a proof $\pi^{\prime}$ shaped as indicated below, such that $h\left(\pi_{1}\right)=h\left(\pi_{1}^{\prime}\right)+1$ and $h\left(\pi_{2}\right)=h\left(\pi_{2}^{\prime}\right)+1$. 


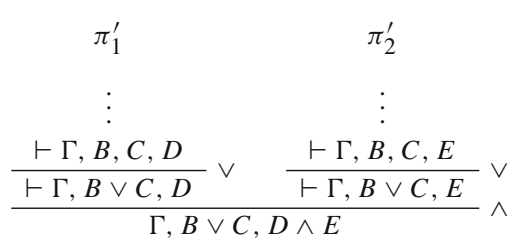

We interchange the two final applications of the logical rules so as to obtain the proof $\rho$ reported below.

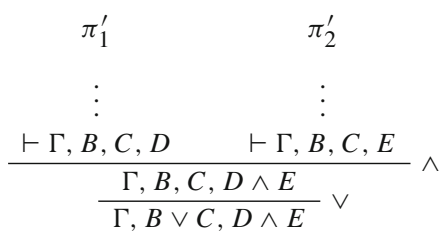

Since $h(\pi)=\max \left(h\left(\pi_{1}\right), h\left(\pi_{2}\right)\right)+1$, we also have $h(\pi)=\max \left(h\left(\pi_{1}^{\prime}\right)+\right.$ $\left.1, h\left(\pi_{2}^{\prime}\right)+1\right)+1$, thence $h(\pi)=\max \left(h\left(\pi_{1}^{\prime}\right), h\left(\pi_{2}^{\prime}\right)\right)+2=h(\rho)$.

- $A \equiv B \wedge C$ and $\pi$ 's last rule is a $\vee$-rule. Let $D \vee E$ be the formula occurring as principal in $\pi$ 's last inference and $\pi_{1}$ the direct subproof of $\pi$ ending in $\vdash$ $\Gamma, B \wedge C, D, E$. By inductive hypothesis, there is a proof $\pi^{\prime}$ shaped as indicated below and such that $h\left(\pi_{1}\right)=\max \left(h\left(\pi_{\langle 1,1\rangle}\right), h\left(\pi_{\langle 1,2\rangle}^{\prime}\right)\right)+1$.

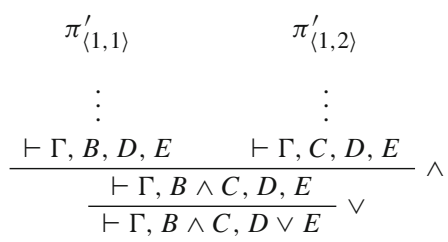

The proof $\rho$ can be obtained from $\pi^{\prime}$ be interchanging the two final applications of the logical rules as indicated below.

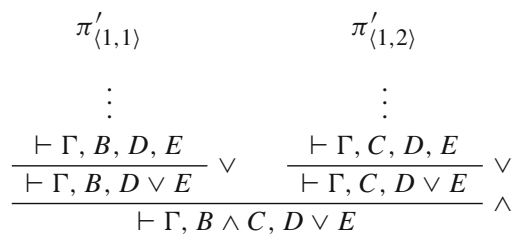

Since, $h(\pi)=h\left(\pi_{1}\right)+1$, we also have $h(\pi)=\max \left(h\left(\pi_{\langle 1,1\rangle}^{\prime}\right), h\left(\pi_{\langle 1,2\rangle}^{\prime}\right)\right)+2=$ $\max \left(h\left(\pi_{\langle 1,1\rangle}^{\prime}\right)+1, h\left(\pi_{\langle 1,2\rangle}^{\prime}\right)+1\right)+1=h(\rho)$.

- $A \equiv B \vee C$ and $\pi$ 's last rule is a $\vee$-rule. Let $D \vee E$ be the formula occurring as principal in $\pi$ 's last inference and $\pi_{1}$ the direct subproof of $\pi$ ending in $\vdash$ $\Gamma, B \vee C, D, E$. By inductive hypothesis, there is a proof $\pi^{\prime}$ shaped as indicated below and such that $h\left(\pi_{1}\right)=h\left(\pi_{1}^{\prime}\right)+1$. 


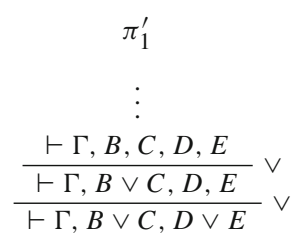

The derivation $\pi^{\prime}$, in turn, can be easily rewritten into the derivation $\rho$ by interchanging the two final applications of the $\vee$-rule as indicated below.

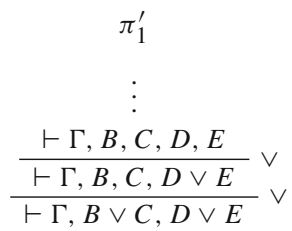

We finally observe that $h(\pi)=h\left(\pi_{1}\right)+1=h\left(\pi_{1}^{\prime}\right)+2=h(\rho)$.

Notation Given a GS4-proof $\pi$ of $\vdash \Gamma, A$ with $\mathcal{C}(A)>0$, we denote with $\mathcal{P}(\pi, A)$ the proof of $\vdash \Gamma, A$ whose last inference is the one introducing $A$ 's principal connective. The proof $\mathcal{P}(\pi, A)$ is intended to be obtained from $\pi$ according to the procedure indicated in the proof of Lemma 3. For $A \equiv B \wedge C$, we indicate with $\mathcal{P}(\pi, A)_{\mathrm{L}}$ and $\mathcal{P}(\pi, A)_{\mathrm{R}}$ the two direct subproofs of $\mathcal{P}(\pi, A)$ ending in $\vdash \Gamma, B$ and $\vdash \Gamma, C$, respectively.

\section{The cut-elimination algorithm}

We call $\mathrm{GS}^{+}$the system obtained by adding to the rules of GS4 the cut-rule in its additive one-sided formulation:

$$
\frac{\vdash \Gamma, A \quad \vdash \Gamma, \bar{A}}{\vdash \Gamma} c u t
$$

When the situation requires it, we will point at specific applications of the cut-rule by adding a subscript $i \in \mathbb{N}$ to the label ' $c u t$ '.

Before going into the details of the cut-elimination algorithm, we need to introduce some key notions to provide a suitable measure for the 'quantity of cut' present in a derivation.

Definition 2 The size of a cut-application

$$
\frac{\vdash \Gamma, C \quad \vdash \Gamma, \bar{C}}{\vdash \Gamma} \mathrm{cut}_{i}
$$

is taken to equal the complexity of the multiset of formulas displayed in one of its premises, i.e., $\left|c u t_{i}\right|=\mathcal{C}(\Gamma, C)=\mathcal{C}(\Gamma, \bar{C})\left(\operatorname{cfr}\right.$. Remark 1). Let $\left\{c u t_{1}, c u t_{2}, \ldots, c u t_{n}\right\}$ be a complete enumeration of the cut-applications occurring in a GS4 ${ }^{+}$-proof $\pi$. The cut-size of $\pi$ is defined as $|\pi|=\max \left\{\left|c u t_{i}\right|+1: 1 \leq i \leq n\right\}$. If $\pi$ is cut-free, then $|\pi|=0$. A cut-application cut $_{i}$ is said to be maximal in $\pi$ whenever $\mid$ cut $_{i}|=| \pi \mid-1$. 
Lemma 4 (Reduction Lemma) Any GS4+-proof $\pi$ of $\vdash \Gamma$ displaying exactly one cutapplication can be turned into a GS4+-proof $\pi^{\prime}$ of the same sequent and such that $\left|\pi^{\prime}\right|<|\pi|$.

Proof We can limit ourselves to considering a proof $\pi$ whose unique cut-application occurs as $\pi$ 's last rule without any loss of generality. Let $\delta$ and $\rho$ be the two direct subproofs of $\pi$ ending in the two premises of the cut-application under consideration:

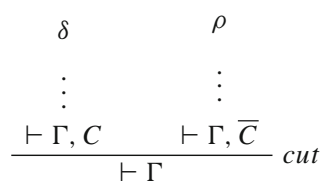

Since $\pi$ contains exactly one cut-application, we immediately have that: $(i)$ both $\delta$ and $\rho$ are cut-free, and $(i i)|\pi|=\mathcal{C}(\Gamma, C)+1=\mathcal{C}(\Gamma, \bar{C})+1$.

If $|\pi|=1$, then the premises of the cut-application are both introduced as instances of the $a x$-rule; say $C \equiv p$, for some atomic sentence $p \in \mathbf{A T}$. It is easy to see that either $\Gamma=\Gamma^{\prime}, p, \bar{p}$ or $\Gamma=\Gamma^{\prime}, q, \bar{q}$ for some $q \in \mathbf{A T}$. Thence, the proof $\pi$ can be simply rewritten as follows:

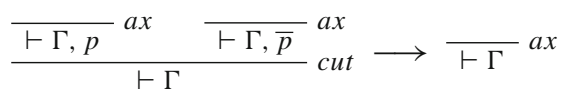

If $|\pi|>1$, we need to proceed by cases and subcases as follows.

[CASE 1] For $\mathcal{C}(C)>0$, we consider the two following subcases according to whether $C$ 's principal connective is a conjunction or a disjunction. Both of them are treated by means of a two-step reduction. The first step (indicated by $\Longrightarrow$ ) is an application of Lemma 3 aiming at permuting downwards the logical rules introducing the principal connective of the cut-formulas $C$ and $\bar{C}$. The second step (indicated by $\longrightarrow$ ) comes as a standard parallel reduction.

[CASE 1.1] If $C \equiv A \wedge B$, then we proceed as follows:

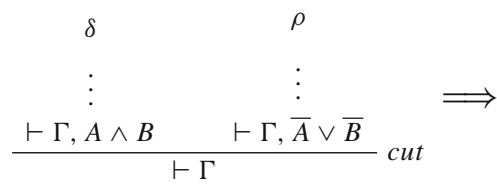

$$
\begin{aligned}
& \mathcal{P}(\delta, A \wedge B)_{\mathrm{L}} \quad \mathcal{P}(\delta, A \wedge B)_{\mathrm{R}} \quad \mathcal{P}(\rho, \bar{A} \vee \bar{B})
\end{aligned}
$$

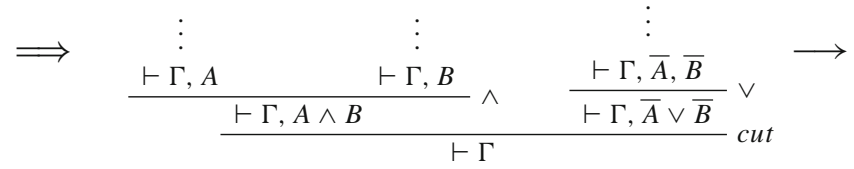

$$
\begin{aligned}
& \mathcal{W}\left(\mathcal{P}(\delta, A \wedge B)_{\mathrm{L}}, \bar{B}\right) \quad \mathcal{P}(\rho, \bar{A} \vee \bar{B})
\end{aligned}
$$

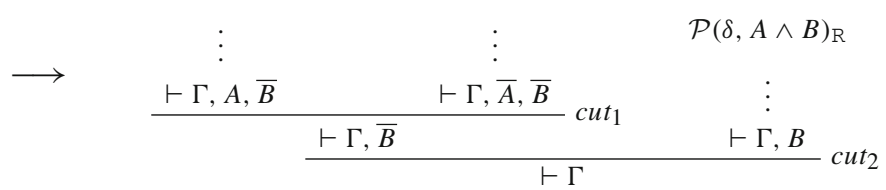


By definition, $|c u t|=\mathcal{C}(\Gamma, A \wedge B),\left|c u t_{1}\right|=\mathcal{C}(\Gamma, A, \bar{B})$, and $\mid$ cut $_{2} \mid=$ $\mathcal{C}(\Gamma, \bar{B})$. Since $\mathcal{C}(B)=\mathcal{C}(\bar{B})$, we can conclude that $\mid$ cut $2|\leq|$ cut $t_{1}|<|$ cut $\mid$.

[CASE 1.2] $C \equiv A \vee B$. Symmetric with respect to the previous one.

[CASE 2] If $\mathcal{C}(C)=0$, since $\mathcal{C}(\Gamma)>0$, there will be a formula $D \in \Gamma$ such that $\mathcal{C}(D)>0$. We need now to distinguish two subcases according to whether $D$ 's principal connective is a conjunction or a disjunction. As for the previous case, we provide a list of two-step reductions. The first reduction $(\Longrightarrow)$ is still an application of Lemma 3 which allows us to permute downward the logical rule introducing the principal connective of $D$. By performing the second step $(\longrightarrow)$ we permute upwards the cut-application under consideration.

[CASE 2.1] $D \equiv A \vee B$

$$
\begin{aligned}
& \begin{array}{cc}
\delta & \rho \\
\vdots & \vdots \\
\frac{\vdash \Gamma, A \vee B, p}{\vdash \Gamma, A \vee B} \quad \vdash \Gamma, A \vee B, \bar{p} \\
\hline \multicolumn{2}{c}{c u t}
\end{array} \Longrightarrow \\
& \mathcal{P}(\delta, A \vee B) \quad \mathcal{P}(\rho, A \vee B) \\
& \Longrightarrow \frac{\frac{\vdash \Gamma, A, B, p}{\vdash \Gamma, A \vee B, p} \vee \frac{\vdash \Gamma, A, B, \bar{p}}{\vdash \Gamma, A \vee B, \bar{p}}}{\vdash \Gamma, A \vee B} \text { cut } \\
& \mathcal{P}(\delta, A \vee B) \quad \mathcal{P}(\rho, A \vee B) \\
& \longrightarrow \frac{\vdash \Gamma, A, B, p \quad \vdash \Gamma, A, B, \bar{p}}{\frac{\vdash \Gamma, A, B}{\vdash \Gamma, A \vee B} \vee}{ }^{-} \vee t_{1}
\end{aligned}
$$

Since $|c u t|=\mathcal{C}(\Gamma, A \wedge B, p)$ and $\left|c u t_{1}\right|=\mathcal{C}(\Gamma, A, B, p)$, we have that $\mid$ cut $_{1}|<|$ cut $\mid$.

[CASE 2.2] $D \equiv A \wedge B$

$$
\begin{aligned}
& \begin{array}{cc}
\delta & \rho \\
\vdots & \vdots \\
\frac{\vdash \Gamma, A \wedge B, p}{\vdash} \quad \vdash \Gamma, A \wedge B, \bar{p} \\
\hline \multicolumn{2}{c}{\text { cut }}
\end{array} \Longrightarrow \\
& \mathcal{P}(\delta, A \wedge B)_{\mathrm{L}} \quad \mathcal{P}(\delta, A \wedge B)_{\mathrm{R}} \quad \mathcal{P}(\rho, A \wedge B)_{\mathrm{L}} \quad \mathcal{P}(\rho, A \wedge B)_{\mathrm{R}}
\end{aligned}
$$

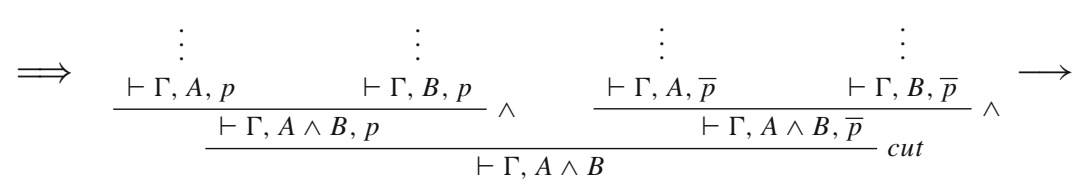




\begin{tabular}{|c|c|c|c|}
\hline $\mathcal{P}(\delta, A \wedge B)_{\mathrm{I}}$ & $\mathcal{P}(\rho, A \wedge B)_{\mathrm{L}}$ & $\mathcal{P}(\delta, A \wedge B)_{\mathrm{R}}$ & $\mathcal{P}(\rho, A \wedge B)_{\mathrm{F}}$ \\
\hline $\begin{array}{c}\vdots \\
\vdash \Gamma, A, p\end{array}$ & $\begin{array}{c}\vdots \\
\vdash \Gamma, A, \bar{p}\end{array}$ & $\begin{array}{c}: \\
\vdash \Gamma, B, p\end{array}$ & $\begin{array}{c}\vdots \\
\vdash \Gamma, B, \bar{p}\end{array}$ \\
\hline & cut $_{1}$ & & \\
\hline
\end{tabular}

In this case we have $|c u t|=\mathcal{C}(\Gamma, A \wedge B, p),\left|c u t_{1}\right|=\mathcal{C}(\Gamma, A, p)$, and $\left|c u t_{2}\right|=$ $\mathcal{C}(\Gamma, B, p)$. Therefore, $\mid$ cut $_{1}|<| c u t \mid$ and $\mid$ cut $_{2}|<| c u t \mid$.

We are now ready to apply the Reduction Lemma to finally prove the following theorem:

Theorem 5 (Hauptsatz) Any GS4 ${ }^{+}$-proof $\pi$ of $\vdash \Gamma$ can be turned into a GS4-proof $\pi^{\prime}$ ending in the same sequent.

Proof The proof is led by a double induction: the principal one is on $|\pi|$, whereas the side induction is on the number of maximal cut-applications. If $|\pi|=1$, then we just keep reducing the topmost cut-applications as indicated in the proof of Lemma 4 till a completely cut-free derivation is achieved.

If $|\pi|>1$, we consider an arbitrarily selected topmost maximal cut-application $c t_{i}$. Let $\delta$ be the subproof of $\pi$ whose last inference is the cut-application under consideration. In particular, let $\delta_{1}$ and $\delta_{2}$ denote the two direct subproofs of $\delta$ ending in the two premises of $c_{i}$ :

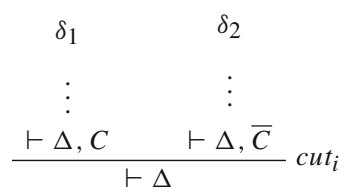

Since cut $_{i}$ occurs as a topmost maximal cut-application, we have $\left|\delta_{1}\right|,\left|\delta_{2}\right|<|\pi|$. By inductive hypothesis, there are two GS4-proofs $\delta_{1}^{\prime}$ and $\delta_{2}^{\prime}$ ending in $\vdash \Delta, C$ and $\vdash \Delta, \bar{C}$, respectively. Consider now the proof $\delta^{\prime}$ obtained from $\delta$ by replacing $\delta_{1}$ with $\delta_{1}^{\prime}$ and $\delta_{2}$ with $\delta_{2}^{\prime}$ :

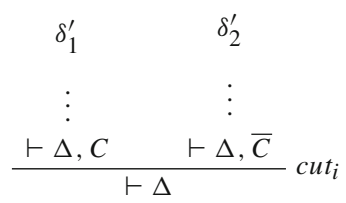

By Lemma 4, there is a GS4 ${ }^{+}$-proof $\delta^{\prime \prime}$ ending in $\vdash \Delta$ and such that $\left|\delta^{\prime \prime}\right|<|\delta|$.

Let $\pi_{1}$ be the proof obtained from $\pi$ by replacing the subproof $\delta$ with $\delta^{\prime \prime}$. The proofs $\pi_{1}$ and $\pi$ end in the same sequent, but $\pi_{1}$ contains one maximal cut-application less than $\pi$. So, it suffices to keep focussing on topmost maximal cut-applications and reiterate the procedure till a proof $\pi_{k}$ of $\vdash \Gamma$ such that $\left|\pi_{k}\right|<|\pi|$ is finally achieved. At this point, our inductive hypothesis guarantees the existence of a cut-free proof $\pi^{\prime}$ ending in $\vdash \Gamma$. 
Remark 2 (First-order logic) The following rules for quantifiers prove reversible in the sense already specified [8].

$$
\frac{\vdash \Gamma, \exists x A, A\left[{ }^{x} / t\right]}{\vdash \Gamma, \exists x A} \exists \quad \frac{\vdash \Gamma, A\left[{ }^{x} / y\right]}{\vdash \Gamma, \forall x A} \forall
$$

Unfortunately, this fact doesn't mean that the technical machinery deployed in this section can be straightforwardly extended so as to prove cut-elimination for the whole first-order system. The reason is simple: for any instance of the $\exists$-rule in which $A(t)$ is non-atomic, $\mathcal{C}\left(\Gamma, \exists x A, A\left[{ }^{x} / t\right]\right)>\mathcal{C}(\Gamma, \exists x A)$.

\section{Bounds}

One of the main advantages of dealing with Kleene's system GS4 lies in the fact that the height of cut-free proofs turns out to be bounded by the complexity of their end-sequent. In particular:

Theorem 6 For any GS4-proof $\pi$ ending in $\vdash \Gamma, h(\pi) \leq \mathcal{C}(\Gamma)+1$.

Proof We proceed by induction on $\mathcal{C}(\Gamma)$. If $\mathcal{C}(\Gamma)=0$, then $\pi$ is just an instance of the $a x$-rule and so $h(\pi)=1$. In case $\mathcal{C}(\Gamma)>0$, we need to distinguish the following two cases.

- The last inference in $\pi$ is an application of the $\wedge$-rule. With $\pi_{1}$ and $\pi_{2}$ we refer to the two direct subproofs of $\pi$ ending in $\vdash \Gamma, A$ and $\vdash \Gamma, B$, respectively. By inductive hypothesis, $h\left(\pi_{1}\right) \leq \mathcal{C}(\Gamma, A)+1$ and $h\left(\pi_{2}\right) \leq \mathcal{C}(\Gamma, B)+1$. Since $h(\pi)=$ $\max \left(h\left(\pi_{1}\right), h\left(\pi_{2}\right)\right)+1$, we can finally conclude that $h(\pi) \leq \mathcal{C}(\Gamma, A \wedge B)+1$.

- The last inference in $\pi$ is an application of the $\vee$-rule. Let $\pi_{1}$ be the direct subproof of $\pi$ ending in $\vdash \Gamma, A, B$. By inductive hypothesis, $h\left(\pi_{1}\right) \leq \mathcal{C}(\Gamma, A, B)+1$. It is also the case that $\mathcal{C}(\Gamma, A \vee B)=\mathcal{C}(\Gamma, A, B)+1$. We then conclude that $h(\pi)=h\left(\pi_{1}\right)+1 \leq \mathcal{C}(\Gamma, A, B)+2=\mathcal{C}(\Gamma, A \vee B)+1$.

A further fact can be also established:

Theorem 7 If $\pi$ and $\rho$ are two GS4-proofs ending in the same sequent $\vdash \Gamma$, then $h(\pi)=h(\rho)$.

Proof We proceed by induction on $\mathcal{C}(\Gamma)$. If $\mathcal{C}(\Gamma)=0$, then $\vdash \Gamma$ is just an instance of the $a x$-rule and so $\pi=\rho$. If $\mathcal{C}(\Gamma)>0$, then there is a multiset $\Gamma^{\prime}$ and a formula $A$ such that $\Gamma=\Gamma^{\prime}, A$ with $\mathcal{C}(A)>0$. We distinguish the following two cases:

- $A \equiv B \wedge C$. Consider the two proofs $\pi^{\prime}$ (the one on the right) and $\rho^{\prime}$ (the one on the left) displayed below.

$$
\begin{array}{cccc}
\mathcal{P}(\pi, B \wedge C)_{\mathrm{L}} & \mathcal{P}(\pi, B \wedge C)_{\mathrm{R}} & \mathcal{P}(\rho, B \wedge C)_{\mathrm{L}} & \mathcal{P}(\rho, B \wedge C)_{\mathrm{R}} \\
\vdots & \vdots & \vdots & \vdots \\
\frac{\vdash \Gamma^{\prime}, B}{\vdash \Gamma^{\prime}, B \wedge C} \wedge & \frac{\vdash \Gamma^{\prime}, B}{\vdash \Gamma^{\prime}, B \wedge C} \wedge
\end{array}
$$


By inductive hypothesis, $h\left(\mathcal{P}(\pi, B \wedge C)_{\mathrm{L}}\right)=h\left(\mathcal{P}(\rho, B \wedge C)_{\mathrm{L}}\right)$ and $h(\mathcal{P}(\pi, B \wedge$ $\left.C)_{\mathrm{R}}\right)=h\left(\mathcal{P}(\rho, B \wedge C)_{\mathrm{R}}\right)$, thence $h\left(\pi^{\prime}\right)=h\left(\rho^{\prime}\right)$. Moreover, by Lemma $3, h(\pi)=$ $h\left(\pi^{\prime}\right)$ and $h(\rho)=h\left(\rho^{\prime}\right)$. The combination of these facts allows us to conclude that $h(\pi)=h(\rho)$.

- $A \equiv B \vee C$. Similar to the previous case.

Funding Open access funding provided by Università degli Studi di Roma Tor Vergata within the CRUICARE Agreement.

Open Access This article is licensed under a Creative Commons Attribution 4.0 International License, which permits use, sharing, adaptation, distribution and reproduction in any medium or format, as long as you give appropriate credit to the original author(s) and the source, provide a link to the Creative Commons licence, and indicate if changes were made. The images or other third party material in this article are included in the article's Creative Commons licence, unless indicated otherwise in a credit line to the material. If material is not included in the article's Creative Commons licence and your intended use is not permitted by statutory regulation or exceeds the permitted use, you will need to obtain permission directly from the copyright holder. To view a copy of this licence, visit http://creativecommons.org/licenses/by/4.0/.

\section{References}

1. Avron, A.: Gentzen-type systems, resolution and tableaux. J. Autom. Reason. 10(2), 265-281 (1993)

2. Gallier, J.H.: Logic for Computer Science: Foundations of Automatic Theorem Proving. Courier Dover Publications, London (2015)

3. Kleene, S.C.: Mathematical Logic. Wiley, New York (1967)

4. Pulcini, G., Varzi, A.: Classical logic through rejection and refutation. In M. Fitting (ed) Landscapes in Logic, vol. 2. College Publications (2021)

5. Schwichtenberg, H.: Proof theory: some applications of cut-elimination. In: Studies in Logic and the Foundations of Mathematics, vol. 90, pp. 867-895. Elsevier (1977)

6. Smullyan, R.M.: First-Order Logic. Courier Corporation, London (1995)

7. W. Tait. Normal derivability in classical logic. In: The Syntax and Semantics of Infinitary Languages, pp. 204-236. Springer (1968)

8. Troelstra, A.S., Schwichtenberg, H.: Basic Proof Theory, vol. 43. Cambridge University Press, Cambridge (2000)

Publisher's Note Springer Nature remains neutral with regard to jurisdictional claims in published maps and institutional affiliations. 